\title{
Inventaire moléculaire d'un écosystème microbien de digestion anaérobie
}

\author{
Jean-Jacques Godon*, Emmanuelle Zumstein, Patrick Dabert, \\ Frédéric Habouzit, René Moletta \\ Laboratoire de biotechnologie de l'environnement, Institut national \\ de la recherche agronomique, Avenue des Étangs, 11100 Narbonne, France
}

\begin{abstract}
Molecular inventory of an anaerobic digestion microbial ecosystem. The bacterial community structure of a fluidised bed reactor fed by vinasses was analysed by molecular identification. After polymerase chain reaction (PCR) amplification, three 16S ribosomal deoxyribonucleic acid (rDNA) clone libraries of Bacteria, Archaea and Procarya populations were established. Community structure was determined by phylogenetic analysis of 556 partial rDNA sequences (about $500 \mathrm{bp}$ long). One hundred thirty-nine operational taxonomic units (OTUs) were found, among which were 133 and 6 from the Bacteria and Archaea domains respectively. Bacterial OTUs are not closely related to all other hitherto-determined sequences (more than $4 \%$ of divergence). The Archaea represent $25 \%$ of the prokaryotic sequences and the most frequent bacterial OTU represents less than $5 \%$ of the bacterial sequences. (C) Inra/Elsevier, Paris

anaerobic digestion / biodiversity / microbial ecology / phylogeny / 16S rRNA

Résumé - L'inventaire moléculaire de la microflore d'un réacteur en lit fluidisé alimenté par des vinasses a été réalisé à partir de trois banques de clones d'ADNr $16 \mathrm{~S}$ (bactéries, archaea et procaryotes) amplifiés par PCR. L'analyse phylogénétique de 556 séquences partielles (environ $500 \mathrm{pb}$ ) décrit la communauté microbienne. Cent trente neuf OTU (Operational Taxonomic Unit) ont été identifiés dont 133 appartiennent au domaine des bactéries et six à celui des archaea. La plupart des séquences sont phylogénétiquement éloignées (plus de $4 \%$ de divergence) des séquences déjà connues. Les archaea représentent $25 \%$ des séquences procaryotes et l'OTU bactérien le plus fréquent moins de $5 \%$ des séquences bactériennes. (c) Inra/Elsevier, Paris
\end{abstract}

digestion anaérobie/ biodiversité/ écologie microbienne/ phylogénie/ ARNr 16S

* Correspondance et tirés à part

Tél : $33(0) 468425154$; fax : $33(0) 468425160$; E-mail : godon@ensam.inra.fr 


\section{INTRODUCTION}

La digestion anaérobie est un procédé biologique simple et efficace pour traiter la pollution carbonée. Cependant notre connaissance de la communauté microbienne impliquée dans la chaîne complexe trophique de la digestion anaérobie reste limitée. En effet, on ne sait isoler et cultiver qu'une fraction de ces microorganismes, l'autre fraction demeurant inconnue. Les techniques moléculaires, notamment celles qui utilisent la molécule de la petite sous-unité des ARN ribosomiques (ARNr 16S), permettent de s'affranchir des techniques culturales pour décrire et suivre un écosystème (Amann et al., 1995). En effet, la séquence de l'ARNr 16S (environ 1500 nucléotides) est spécifique de chaque organisme et de son évolution depuis un ancêtre commun. De plus, le grand nombre de séquences actuellement disponibles (plus de 6000 séquences de procaryotes) permet de dessiner un vaste arbre phylogénétique des microorganismes et d'y placer chaque nouvelle séquence. A partir des alignements de séquences il est ensuite possible de choisir des sondes nucléiques qui permettront de cibler une espèce, un genre ou un phylum. Il devient alors possible théoriquement de suivre cette espèce, ce genre ou ce phylum dans un écosystème complexe sans jamais l'avoir isolé en culture pure.

Des sondes nucléiques ont déjà été utilisées sur un digesteur anaérobie (Raskin et al., 1994). Cependant ces sondes ont été créées à partir des séquences d'ARNr 16S de microorganismes ou de groupes de microorganismes précédemment isolés en culture pure. Dans ce cas, la puissance de ces outils demeure encore limitée par les contingences de l'isolement. L'inventaire moléculaire apparaît donc comme un outil nouveau pour décrire un écosystème microbien donné et suivre son évolution.

Le travail présenté dans cet article est l'inventaire moléculaire, aussi exhaustif que possible, des microorganismes composant le biofilm d'un digesteur anaérobie à lit fluidisé.

\section{MATÉRIEL ET MÉTHODES}

\subsection{Réacteur anaérobie étudié}

Le réacteur étudié est un lit fluidisé, alimenté par des vinasses provenant d'une distillerie locale. Ce polluant industriel est le résidu de la distillation de vin et de marc. Il présente l'avantage d'être quasiment stérile, évitant ainsi que des microorganismes en «transit» ne perturbent l'image de l'écosystème. Le réacteur est tubulaire avec un volume de $0,6 \mathrm{~L}$. Le support colonisé par le biofilm est de la pouzzolane (poudre de roche volcanique) et la fluidisation produit une extension du volume de $25 \%$. Le carbone organique total (COT) de la vinasse est en moyenne de $10 \mathrm{~g} \mathrm{~L}^{-1}$ et la demande chimique en oxygène (DCO) de $25 \mathrm{~g} \mathrm{~L}^{-1}$. La charge organique appliquée est de $5,5 \mathrm{~g}$ de $\mathrm{DCO} / \mathrm{L} / \mathrm{j}$. Le pH et la température sont maintenus respectivement autour de 7 et de $35^{\circ} \mathrm{C}$. Les quantités de COT et d'acides gras volatils se maintiennent régulièrement respectivement autour de $800 \mathrm{mg} \mathrm{L}^{-1}$ et $250 \mathrm{mg} \mathrm{L}^{-1}$. Le réacteur a été inoculé à $100 \%$ du volume à partir de boues issues d'un réacteur similaire (à lit fluidisé) 
alimenté avec le même substrat et qui fonctionnait depuis plus d'un an en continu (Buffière et al., 1995). L'échantillon analysé a été collecté 40 jours après l'inoculation.

\subsection{Méthodes pour un inventaire moléculaire}

Les étapes sont successivement : (i) l'extraction de l'ADN total des microorganismes composant l'écosystème; (ii) l'amplification par PCR de la région de l'ADNr $16 \mathrm{~S}$ grâce à des amorces spécifiques choisies dans les régions conservées de la molécule; (iii) l'isolement par clonage des molécules ainsi amplifiées; (iv) le séquençage du fragment d'ADN cloné. Toutes ces techniques ont été précédemment décrites (Godon et al., 1997 ; Sambrook et al., 1989).

Trois banques d'ADNr 16S ont été réalisées : ADNr 16S archaea total ( $E$. coli position 6 à 1509 (Brosius et al., 1981)), ADNr 16S bactérien total (E. coli position 8 à 1509 ) et $\mathrm{ADNr} 16 \mathrm{~S}$ procaryote partiel (E. coli position 778 à 1509). Une séquence partielle d'environ $500 \mathrm{pb}$ qui représente un tiers de la molécule $\mathrm{ARNr} 16 \mathrm{~S}$ a été réalisée pour chaque clone $(E$. coli position 812 à 1307). Chaque séquence a été comparée avec les autres séquences produites et avec les séquences d'ARNr $16 \mathrm{~S}$ disponibles dans les bases de données Genbank et Ribosome Database Project (Maidak et al., 1994).

\section{RÉSULTATS ET DISCUSSION}

\subsection{Diversité de la microflore et concept d'OTU}

Pour analyser la diversité, la notion d'OTU (Operational Taxonomic Unit), équivalent moléculaire de l'espèce, a été utilisée pour regrouper les séquences les plus proches (Moyer et al., 1994). Ce regroupement se heurte à plusieurs problèmes. D'une part, les 556 clones analysés présentent un continuum de divergence de 0 à $30 \%$ et, d'autre part, des séquences d'ARNr $16 \mathrm{~S}$ différentes peuvent provenir soit de deux microorganismes différents, soit de deux copies différentes d'ARNr $16 \mathrm{~S}$ issues du même chromosome. Par exemple, chez Haloarcula marismortui deux copies de l'ARNr $16 \mathrm{~S}$ présentent $5 \%$ de divergence (Mylvaganam et Dennis, 1992). Ainsi, il est délicat de fixer un seuil de divergence entre OTU et selon le seuil fixé, le nombre d'OTU peut varier fortement (figure 1).

Arbitrairement, les 556 séquences ont été regroupées sur les bases de $96 \%$ de similarité au sein d'un OTU formant ainsi 139 OTU. Ce nombre diffère sensiblement de la figure 1 car certains OTU comprennent des séquences qui divergent de plus de $4 \%$ mais qui sont reliées par un continuum de séquences divergeant entre elles de moins de $4 \%$.

Les clones bactériens représentent la plus grande diversité avec 133 OTU identifiés parmi 460 clones bactériens analysés alors que seulement six OTU archaea ont été identifiés parmi 96 clones analysés. Les séquences des 139 OTU sont disponibles dans les bases de données EMBL, Genbank et DDBJ sous les numéros d'accession : U81640 à U81778. 


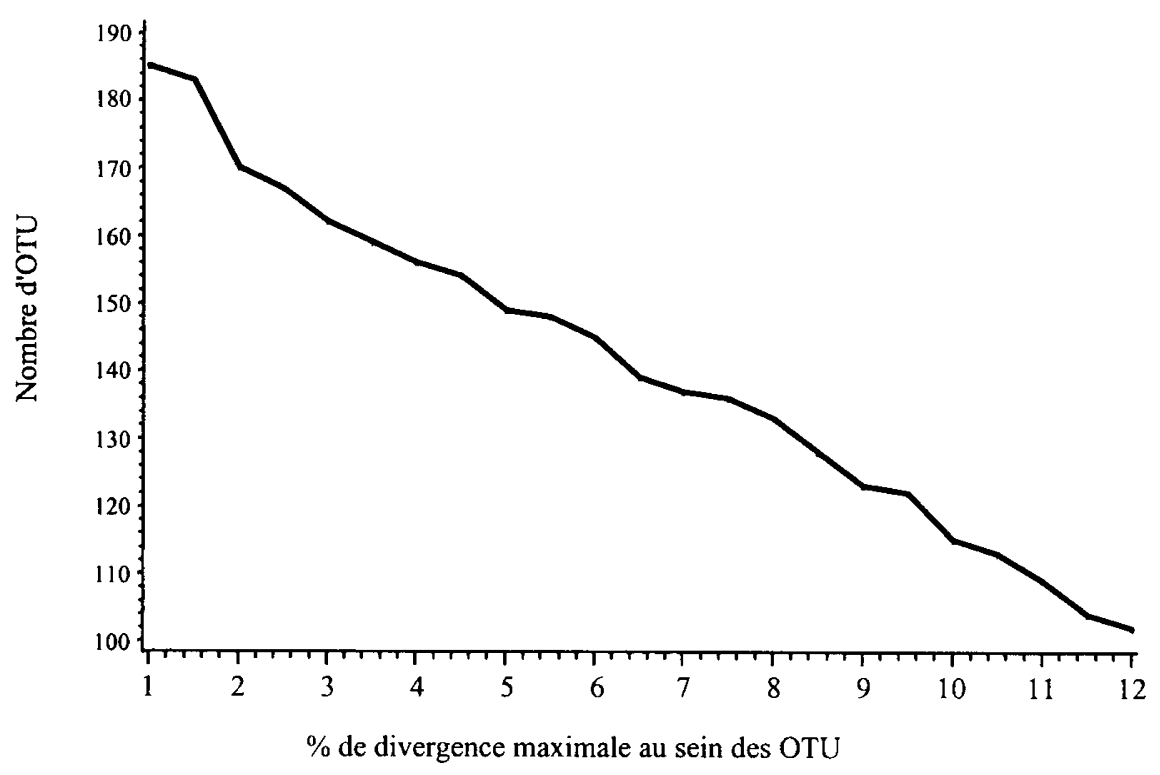

Figure 1. Nombre d'OTU en fonction du seuil de divergence au sein des OTU.

\subsection{Diversité phylogénétique (approche qualitative)}

La distribution phylogénétique des 139 OTU parmi les phylums majoritaires est précisée sur la figure 2. $79 \%$ des OTU appartiennent à trois phylums majeurs des bactéries : les protéobactéries, les bactéries Gram positive à bas GC \% et le groupe Cytophaga-Flexibacter-Bacteroides (CFB). Les autres OTU sont répartis parmi des phylums mineurs et certains peuvent difficilement être affiliés à un phylum connu.

Aucun $\mathrm{ADNr} 16 \mathrm{~S}$ bactérien séquencé a moins de $3 \%$ de divergence avec une séquence présente dans les bases de donnés. Les séquences d'ADNr $16 \mathrm{~S}$ des archaea méthanogènes sont en revanche, quasiment similaires (moins de $3 \%$ ) avec celles de Methanosarcina barkeri $(1,2 \%)$, Ms. frisius $(0,2 \%)$ et Methanobacterium formicicum (3\%). À l'inverse, les trois autres OTU archaea sont très atypiques. La figure 3 présente le positionnement phylogénétique des OTU procaryotes.

\subsection{Répartition des clones (approche quantitative)}

L'analyse phylogénétique présentée dans le chapitre précédent décrit la diversité de la population sur la base des OTU. Dans ce chapitre les résultats sont présentés au niveau des clones et estiment leur répartition au sein de la population.

Parmi les clones analysés, 460 et 96 appartiennent respectivement aux domaines des bactéries et des archaea, la répartition des clones bactériens à partir des banques bactéries et procaryotes étant similaire. La figure 4 indique la fréquence des clones dans les groupes phylogénétiques. 
Archaea methanogene $2 \%$ Autres Archaea $2 \%$

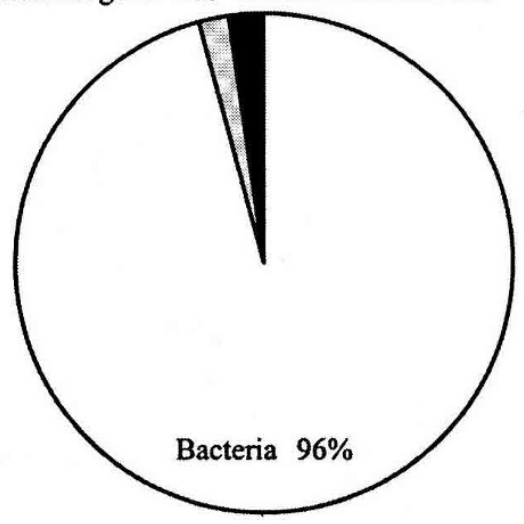

B

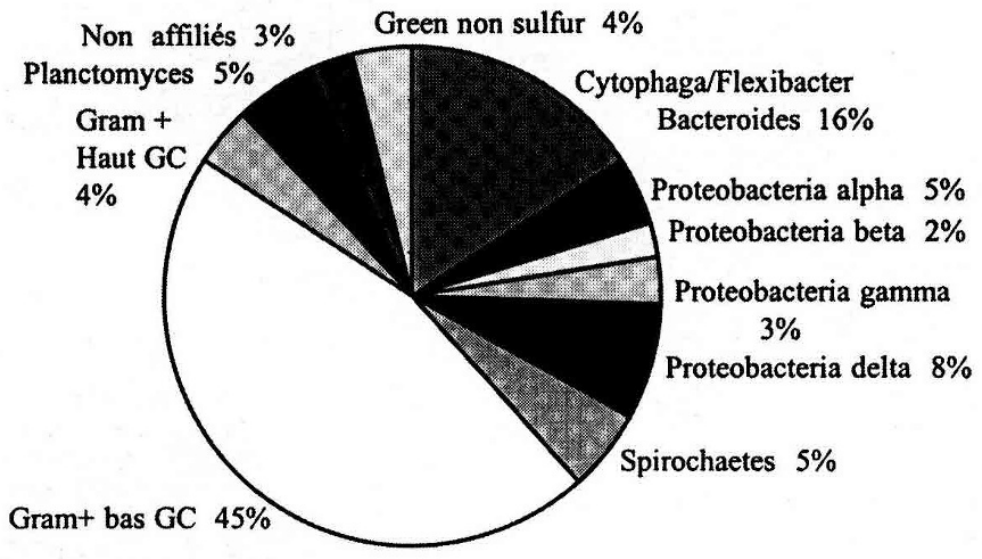

Figure 2. Distribution des OTU. A : OTU procaryotes (bactériens et archaea). B : OTU bactériens.

Parmi les OTU bactérien, le plus fréquent comprend 22 clones ce qui correspond à $5 \%$ de la totalité des clones bactériens. Quatorze OTU comprennent neuf clones ou plus et ont une fréquence supérieure à $2 \%$. Dix sept OTU comprennent de cinq à huit clones et ont une fréquence entre 1 et $2 \%$. Trente-neuf OTU comprennent deux à quatre clones et ont une fréquence entre 0,2 et $0,9 \%$. Enfin, soixante OTU comprennent seulement un clone et ont une fréquence inférieure à $0,2 \%$.

Le plus fréquent des OTU archaea comprend 43 clones ce qui correspond à $44 \%$ des clones archaea et à $11 \%$ des clones procaryotes. 


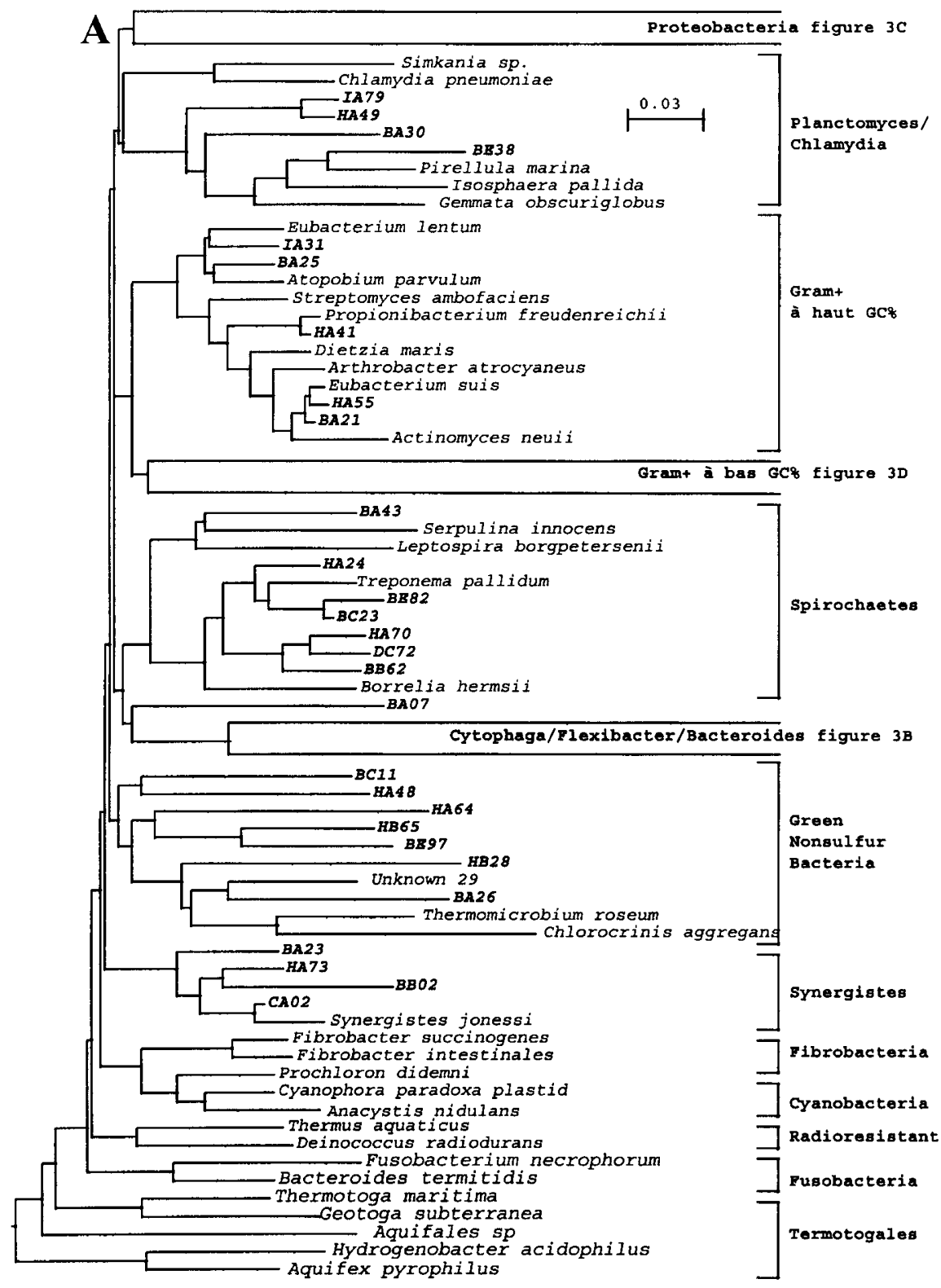

Figure 3a. 


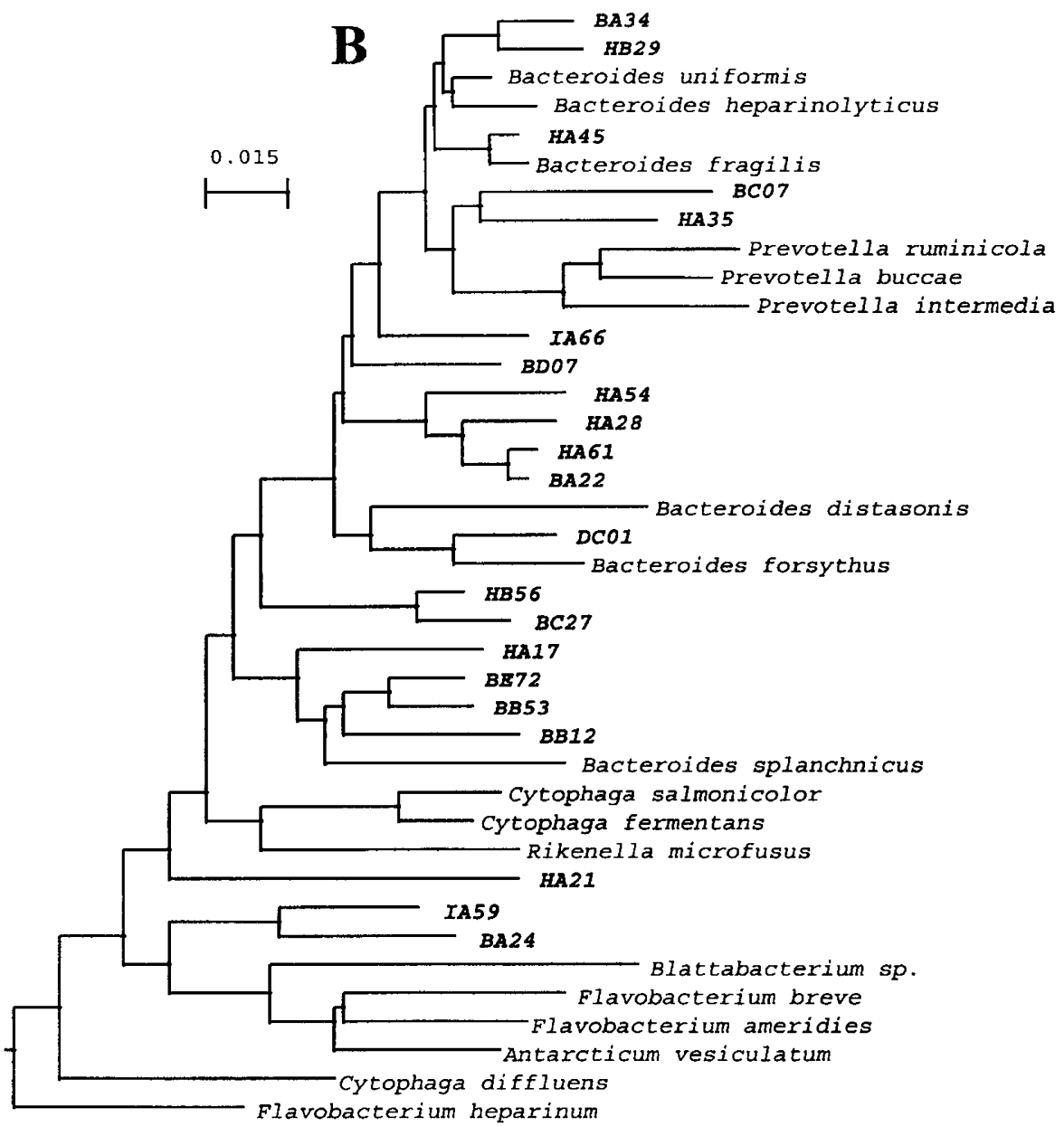

Figure 3b. 


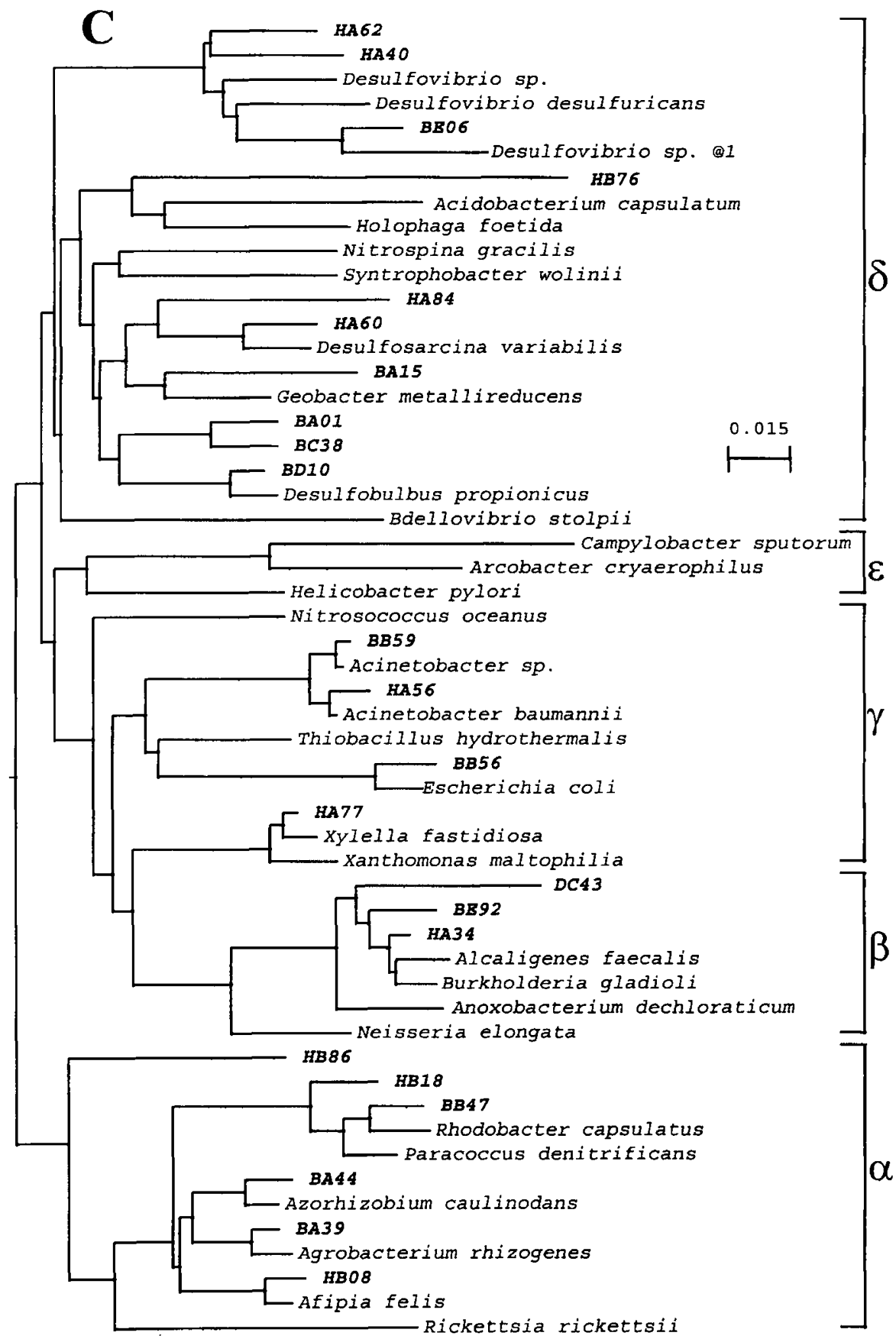

Figure 3c. 


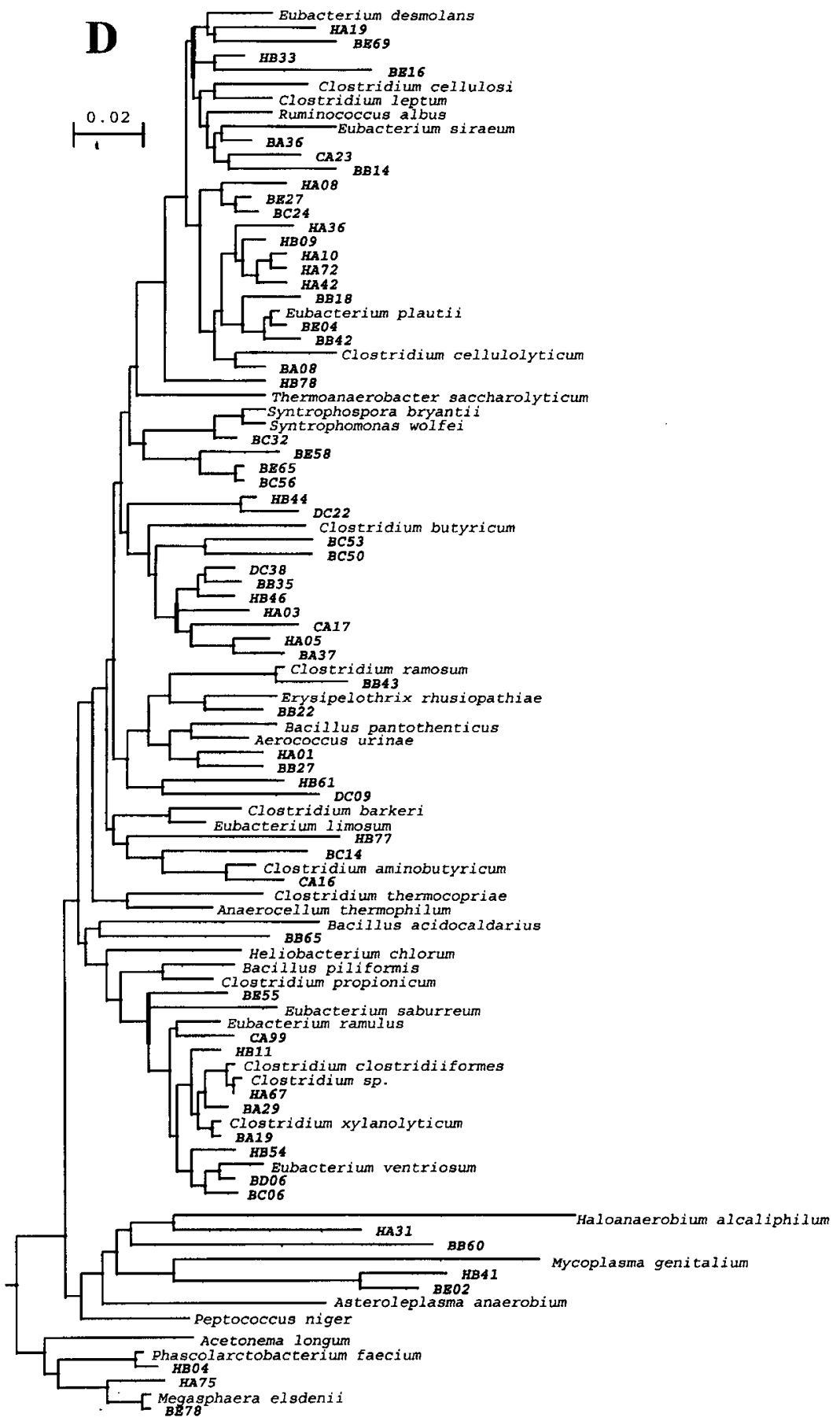

Figure 3d. 


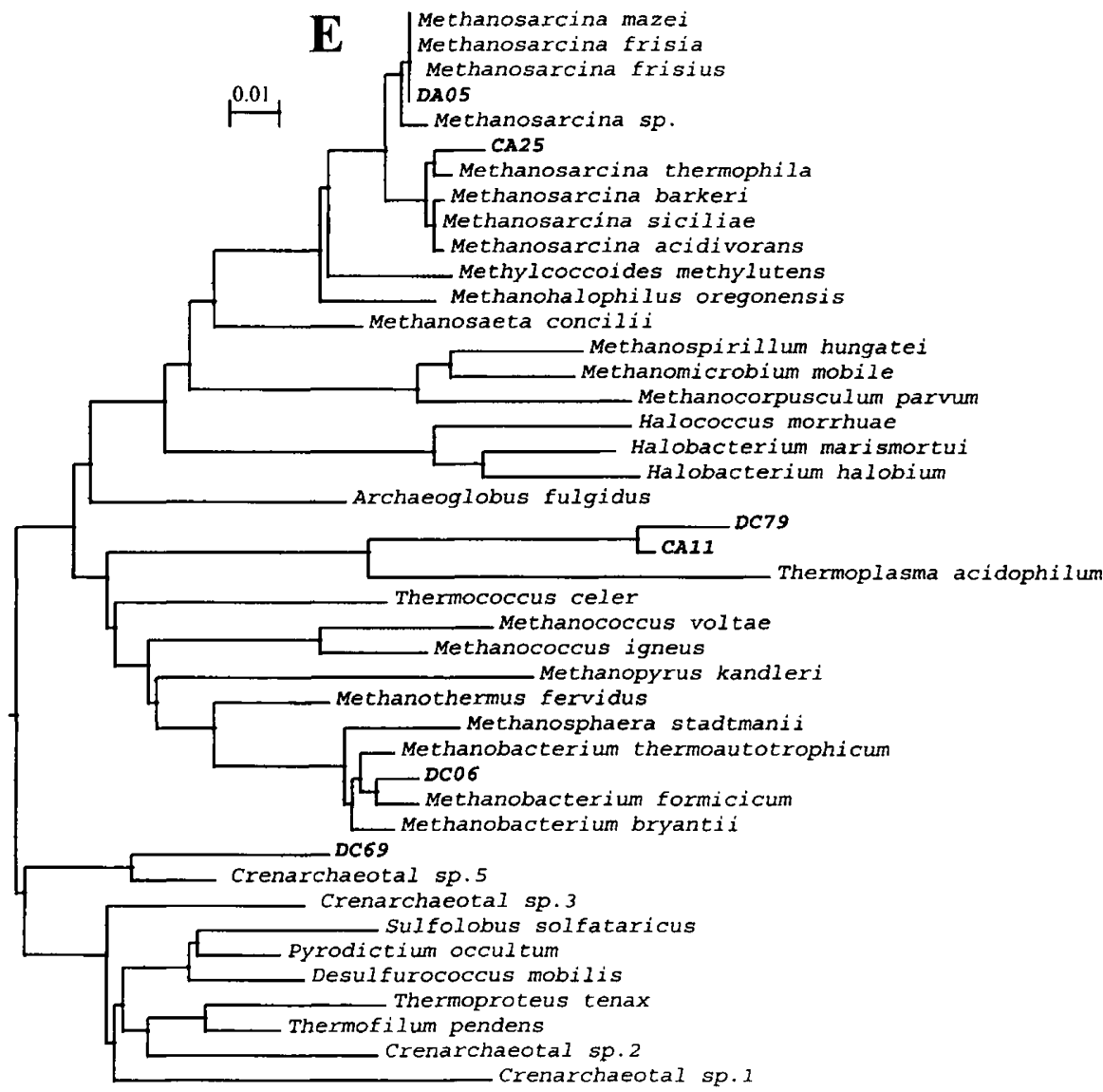

Figure 3. Phylogénie moléculaire entre les OTU décrits et les séquences les plus proches des bases de données, basée sur des séquences partielles de l'ADNr 16S (environ $500 \mathrm{pb}$ ). Les matrices de distance et l'arbre phylogénétique ont été établis grâce aux algorithmes de Jukes-Cantor (Jukes et Cantor, 1969) et neighbor-joining (Saitou et Nei, 1987). La distance entre les séquences est la somme des lignes horizontales et l'échelle représente le nombre de changements de nucléotides par longueur. A : OTU appartenant à des groupes minoritaires du domaine des bactéries. La séquence de l'archaea $M$. sarcina permet d'enraciner l'arbre. B : OTU appartenant au groupe des Cytophaga, Flexibacter, Bacteroides. La séquence de la bactérie Chlorobium vibrioforme permet d'enraciner l'arbre. C : OTU appartenant au groupe des protéobactéries. La séquence de la bactérie Bacillus subtilis (Gram positive à bas GC \%) permet d'enraciner l'arbre. D : OTU appartenant au groupe des bactéries Gram positive à bas GC \%. La séquence de la bactérie Streptomyces ambofaciens (Gram positive à haut GC \%) permet d'enraciner l'arbre. E : OTU appartenant au domaine des archaea. La séquence de la bactérie $E$. coli permet d'enraciner l'arbre.

\subsection{Image de la population}

Cet écosystème apparemment stable et clos est composé de plus de 139 microorganismes différents classés comme procaryotes. Cet inventaire n'est 
A

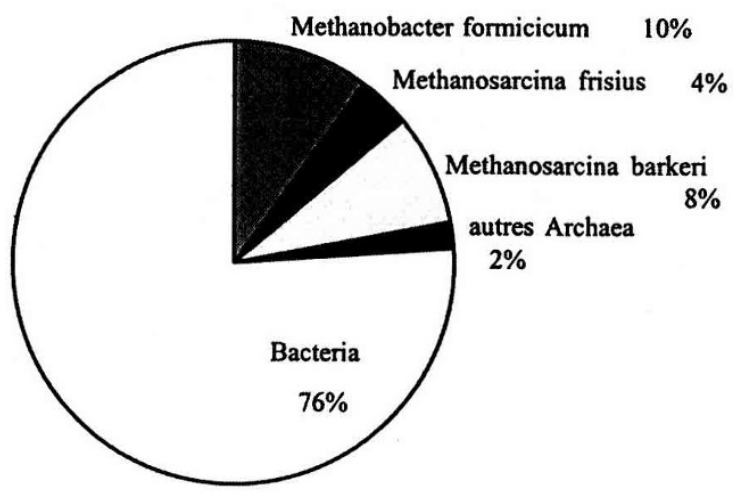

B

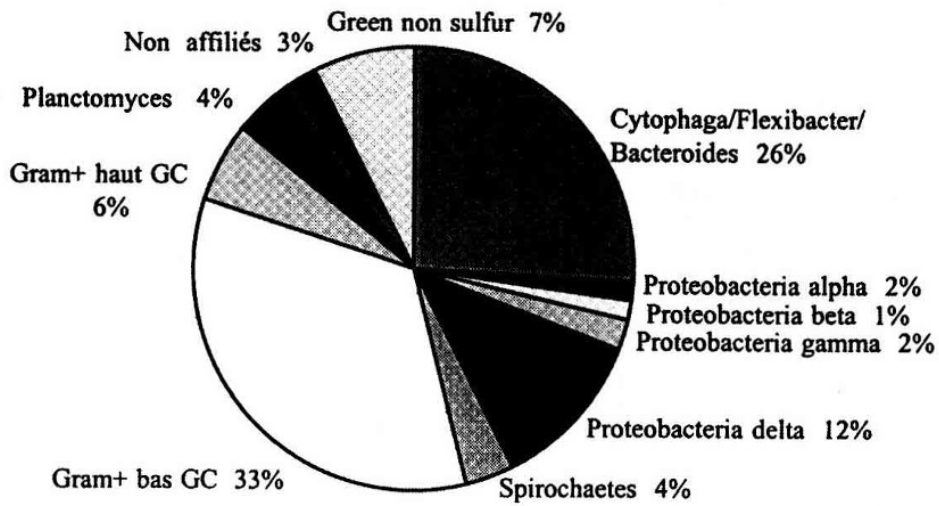

Figure 4. Répartition des clones. A : Clones procaryotes (bactéries et archaea). $\mathrm{B}$ : Clones bactériens.

pas exhaustif, le nombre total d'espèces demeure inconnu et encore hors de portée. Après l'analyse de 460 clones, la découverte de nouveaux OTU est de moins en moins fréquente : à ce point de l'analyse, on identifie un nouvel OTU pour dix nouveaux clones séquencés (figure 5). On peut estimer que les OTU ayant une fréquence supérieure à $1 \%$ donc majoritaires dans l'écosystème ont été décris.

\subsection{Biais due à la méthode}

La pertinence des résultats dépend du biais introduit par les techniques employées. Plusieurs paramètres peuvent modifier les résultats : le contenu en GC de l'ARNr 16S, des sensibilités différentes des parois cellulaires à la lyse, certaines spécificités de la Taq polymérase, la spécificité des amorces, la 


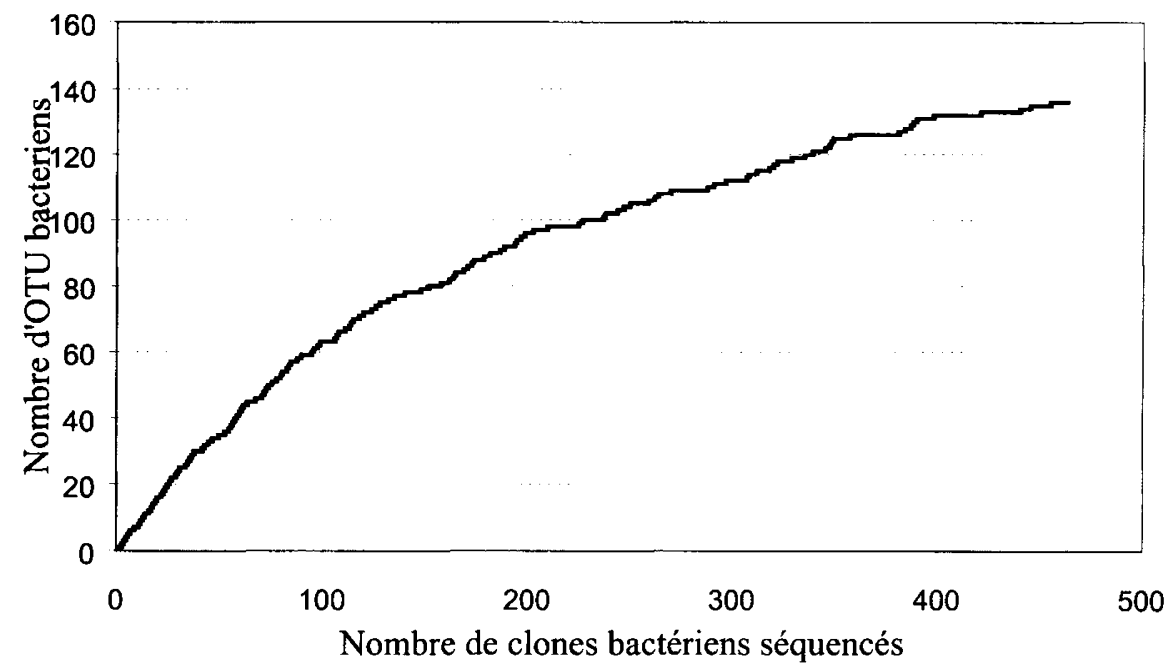

Figure 5. Estimation de la diversité bactérienne. Apparition de nouveaux OTU lors de l'analyse de 460 séquences d'ARNr $16 \mathrm{~S}$ bactériens.

formation de molécules chimères ou le nombre de copies des gènes codant pour l'ARNr 16S. Cependant la grande diversité observée modère l'impact de ces facteurs sur les résultats. Le problème le plus gênant demeure la formation de molécules chimères pendant l'amplification par PCR d'une population d'ADN, une molécule chimère d'ADNr étant composée de fragments d'ADNr issus d'organismes différents. Cette molécule est en effet difficilement identifiable. Ce délicat problème peut être partiellement résolu par la comparaison d'un nombre important de séquences provenant de PCR indépendantes. Vingt-six chimères ont ainsi été détectées et retirées des clones bactériens $(5,4 \%)$.

Ainsi la fréquence des microorganismes dans l'écosystème fournie par cette approche n'est probablement pas l'exacte image de la réalité mais ces résultats peuvent être utilisés comme point de départ pour une analyse plus poussée de l'écosystème.

Ce travail donne une description microbiologique qualitative et quantitative de la biodiversité d'un écosystème de digestion anaérobie en utilisant la séquence des $\mathrm{ADNr} 16 \mathrm{~S}$. Un très grand nombre d'organismes nouveaux sont présents au sein de cet écosystème commun. Cette diversité apparente doit être modérée car seulement quelques organismes majoritaires semblent représenter l'essentiel de la population. Ces résultats sont compatibles avec la description de l'écosystème de digestion anaérobie par des méthodes classiques. L'identification moléculaire par l'ADNr $16 \mathrm{~S}$ retrouve des membres des genres ou des taxons attendus pour lesquels la fonction dans la chaîne trophique est connue : exemple Bacteroides, Eubacterium, Clostridium, Proteobacteria delta (bactérie sulfatoréductrice), syntrophomonas, archaea méthanogènes, etc. D'autre part, cette approche indépendante de l'isolement des microorganismes, révèle des membres appartenant à des genres dont la fonction est inconnue comme les Spirochaetes, les «green non-sulfur», les Planctomyces et les Archaea non méthanogènes. 
Ces résultats soulèvent le problème de la mesure de la diversité chez les procaryotes. Le grand nombre de séquences analysées aborde par une approche moléculaire (divergence de la molécule d'ARN 16S) la question de "l'espèce». Or, des liens cohérents restent encore à établir entre une vision moléculaire et une vision fonctionnelle de «l'espèce».

\section{REMERCIEMENTS}

Nous tenons à remercier P. Bellenand pour sa contribution à l'analyse des résultats.

\section{RÉFÉRENCES}

Amann R.I., Ludwig W., Schleifer K.H., Phylogenetic identification and in situ detection of individual microbial cells without cultivation, Microbiol. Rev. 59 (1995) 143-169.

Brosius J., Dull T.J., Sleeter D.D., Noller H.F., Gene organisation and primary structure of a ribosomal RNA operon from Escherichia coli, J. Mol. Biol. 148 (1981) $107-127$.

Buffière P., Moletta R., Fonade C., Continuous operations of fluidized bed bioreactor for anaerobic digestion : residence time effect on degradation kinetics, Biotechnol. Lett. 17 (1995) 833-838.

Godon J.-J., Zumstein E., Dabert P., Habouzit F., Moletta R., Microbial SSU rDNA diversity in an anaerobic digestor, Appl. Environ. Microbiol. 63 (1997) 28022813.

Jukes T.H., Cantor C.R., Evolution of protein molecules. In : Munro H.N. (Ed.), Mammalian protein metabolism, Academic Press, New York, 21-132, 1969.

Maidak B.L., Larsen N., McCaughey M.J., Overbeek R., Olsen G.J., Fogel K., Blandy J., Woese C.R., The Ribosomal Database Project, Nucleic Acids Res. 22 (1994) 3485-3487.

Moyer C.L., Dobbs F.C., Karl D.M., Estimation of diversity and community structure through restriction fragment length polymorphism distribution analysis of bacterial $16 \mathrm{~S}$ rRNA genes from a microbial mat at an active hydrothermal vent system, Loihi Seamount, Hawaii, Appl. Environ. Microbiol. 60 (1994) 871-879.

Mylvaganam S., Dennis P., Sequence heterogeneity between the two genes encoding 16S rRNA from the halophilic archaebacterium Haloarcula marismortui, Genetics 130 (1992) 399-410.

Raskin L., Poulsen L.R., Noguera D.R., Rittmann B.E., Stahl D.A., Quantification of methanogenic groups in anaerobic biological reactors by oligonucleotide probe hybridisation, Appl. Environ. Microbiol. 60 (1994) 1241-1248.

Saitou N., Nei M., The neighbor-joining method : a new method for constructing phylogenetic trees, Mol. Biol. Evol. 4 (1987) 406-425.

Sambrook J., Fritsch E.F., Maniatis T., Molecular cloning : A laboratory manual, Cold Spring Harbor Laboratory Press, Cold Spring Harbor, NY, 1989. 